\title{
Experimental and Simulation Modeling Studies of Magnetic Properties of Ni-Zn Ferrite Cores under DC Bias

\author{
H. OSHIMA ${ }^{\text {a) }}$, Y. UEHARA ${ }^{\text {b) }}$, K. SHIMIZU ${ }^{\text {b) }}$, K. INAGAKI ${ }^{(\text {) }}$, A. FURUYA $^{\text {b) }}$, J. FUJISAKI ${ }^{\text {b) }}$, M. SUZUKI ${ }^{\text {d) }}$, \\ K. KAWANO ${ }^{\text {d) }}$, T. MIFUNE ${ }^{\text {e) }}$, T. MATSUO ${ }^{\mathrm{e}}{ }^{\text {, }}$ K. WATANABE ${ }^{\text {f) }}$, and H. IGARASHI $^{\mathrm{g}}$
}

a) Fujitsu Laboratories Limited, 10-1 Morinosato-Wakamiya, Atsugi 243-0197, Japan

b) Fujitsu Limited, 4-1-1 Kamikodanaka, Nakahara-ku, Kawasaki 211-8588, Japan

c) Fujitsu Advanced Technologies Limited, 4-1-1 Kamikodanaka, Nakahara-ku, Kawasaki 211-8588, Japan

d) Taiyo Yuden Corporation Limited, 5607-2 Nakamuroda-machi, Takasaki-shi, Gunma 370-3347, Japan

e) Department of Electrical Engineering, Kyoto University, Kyoto 615-8510, Japan

f) Department of Information and Electronic Engineering, Muroran Institute of Technology, Hokkaido 050-8585, Japan

g) Graduate School of Information Science and Technology, Hokkaido University, Sapporo 060-0814, Japan

\begin{abstract}
$\mathrm{Ni}-\mathrm{Zn}$ ferrite cores under dc bias are experimentally characterized, and the results are compared with those of simulation. Hysteretic behavior of a toroidal core at high frequencies and the effect of dc bias are well reproduced by simulation with a hysteresis model that we have developed recently, a grain magnetics (GM) model. The inductance and core loss of a wirewound inductor under high-frequency excitation with dc bias are also measured. We show that the finite element analysis with the hysteresis model provides simulation results close to the experimental data of the inductor.
\end{abstract}

Key Words: Ni-Zn ferrite, Inductors, Core loss, Hysteresis model, Finite element analysis

\section{INTRODUCTION}

Accurate prediction of core loss in inductive components, such as inductors in de/dc converters, is crucial for future computer-aided device design. It is, however, still quite challenging. In particular, the influence of dc bias is not entirely clarified both from experimental and simulation points of view [1,2]. The purposes of this study are therefore as follows: (i) precise measurement of inductor ferrite cores under dc bias, (ii) hysteresis modeling to reproduce their hysteretic behavior, and (iii) finite element analysis (FEA) with the hysteresis model for accurate calculations of inductance and core loss. In this study, we focus our attention to Ni-Zn ferrite, which has been widely used for high-frequency application [3].

\section{MEASUREMENT OF FERRITE CORES UNDER DC BIAS}

For measurement of magnetic properties of ferrite cores under various conditions, we have developed a measurement system that enables us to apply dc-biased sinusoidal electric current up to $1 \mathrm{MHz}$ through a single primary winding wire. A schematic diagram of the system is depicted in Fig. 1. From the ac and dc current sources of the system, ac and dc currents are applied in parallel to the primary winding. The capacitors and low-pass filters are inserted in the circuits to insulate dc and ac current from the other source, respectively.
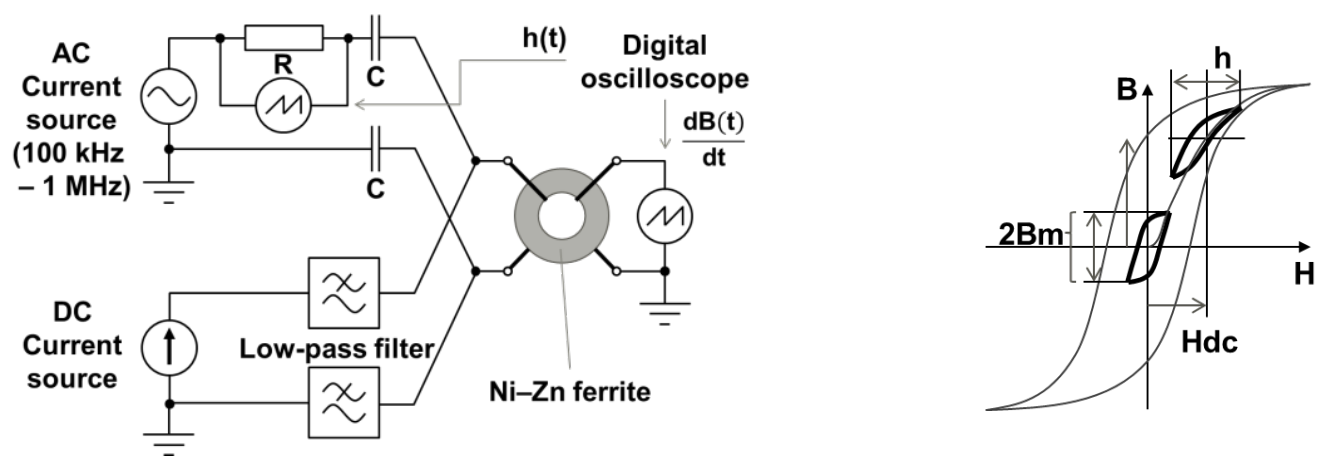

Fig. 1 Measurement system for ferrite cores.

Fig. 2 Minor $B-H$ loops with and without de bias. 
For $B$ - $H$ loop measurement, we use a toroidal core of $\mathrm{Ni}-\mathrm{Zn}$ ferrite, $\mathrm{Ni}_{0.49} \mathrm{Zn}_{0.49} \mathrm{Co}_{0.02} \mathrm{Fe}_{1.90} \mathrm{O}_{\mathrm{x}}$ [3]. AC magnetic field $h$ and magnetic flux density $B$ are calculated from the voltages across the shunt resistance and the secondary winding, respectively. Minor loops with and without dc bias are shown schematically in Fig. 2. Hereafter, $B_{\mathrm{m}}$ is the amplitude, and $H_{\mathrm{dc}}$ and $h$ stand for dc bias and ac magnetic field, respectively. Magnetic field $H$ is the sum of $H_{\mathrm{dc}}$ and $h$. Figure 3 shows some of our experimental results. The frequency dependence in Fig. 3 presents that the loops become wider when the frequency increases, probably because of the friction of the domain wall motion [3]. The dc bias dependence in Fig. 3 shows that the loops become stretched and asymmetric when we increase the dc bias. For the inductance and core loss measurement, a simple wirewound inductor of the same $\mathrm{Ni}-\mathrm{Zn}$ ferrite material is used. The inductance and core loss are calculated from the current and the voltage drop at the winding of the inductor. The results will be shown in Section IV.
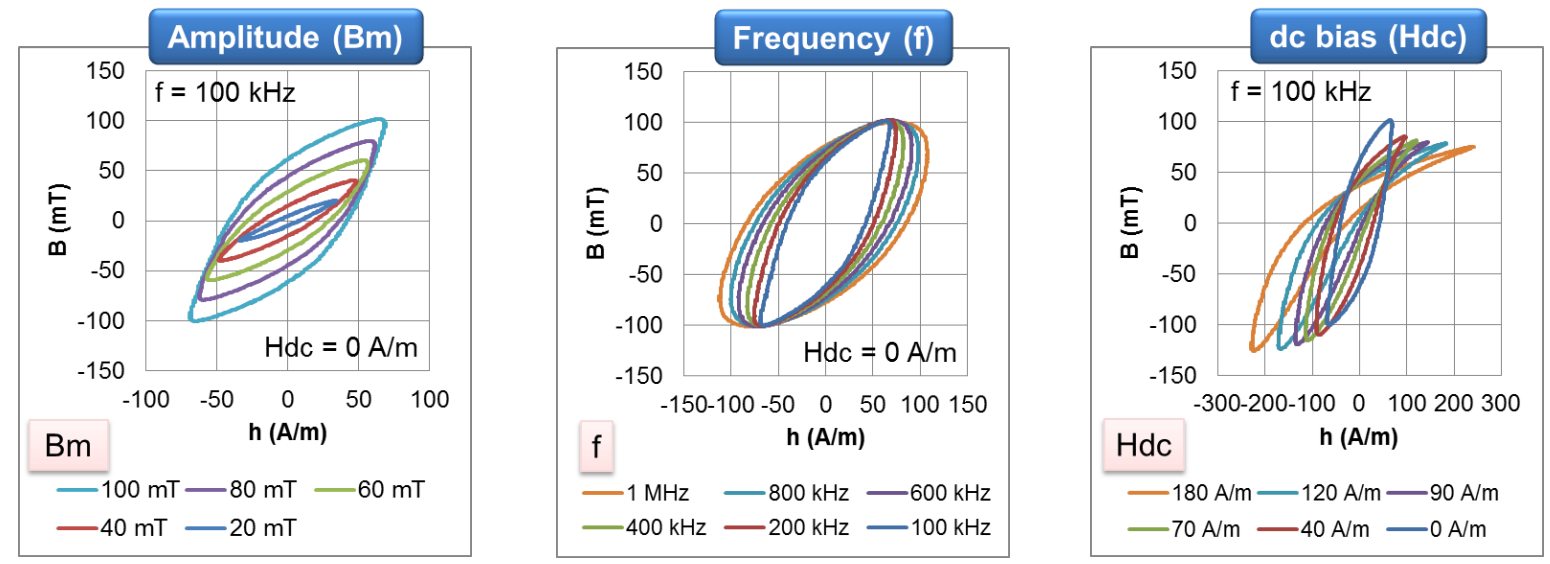

Fig. 3 Experimental results for minor $B-H$ loops under various conditions.

\section{HYSTERESIS MODELING}

The next step is to model the hysteretic behavior. Phenomenological models, such as Preisach [4] and Jiles-Atherton [5] models, are known to be capable of reproducing various types of hysteresis curves. They are, however, empirical and/or mathematical. On the other hand, physical models, such as Stoner-Wohlfarth (SW) model [6] and micromagnetics based on it [7], are more directly connected with the real physical properties. The problem of the physical models is that they require too much computing power for simulation of bulk material.

In this study, we propose a new hysteresis model based on the physical model for bulk ferrite material. Here, grains are represented by single domain SW particles [8] (Fig. 4), which greatly reduces required computing power. However, in reality, each grain has magnetic domain structures and is not a single domain particle. To include the effects of magnetic domain structures, therefore, we suggest four assumptions below and term it a "grain magnetics (GM)" model [9]. First, we assume drastic reduction and normal distribution of the crystalline anisotropy field $\left(H_{\mathrm{k}}\right.$ with a standard deviation $\left.\sigma_{\mathrm{Hk}}\right)$, because the magnetization reversal fields of multidomain grains are generally much reduced and statistically dispersed. Second, we include an effective field $\left(H_{\mathrm{w}}\right)$ due to the friction of domain-wall motion, which is proportional to $-\mathrm{d} M / \mathrm{d} t$. Third, we include a small demagnetization field $\left(H_{\mathrm{d}}\right)$ due to grain structures, which is proportional to $-M$. Fourth, we neglect exchange and stray fields between the magnetizations because of the large grain size. Magnetization distributions are calculated with this model by minimizing total magnetic energy using the Landau-Lifshitz-Gilbert (LLG) equation.
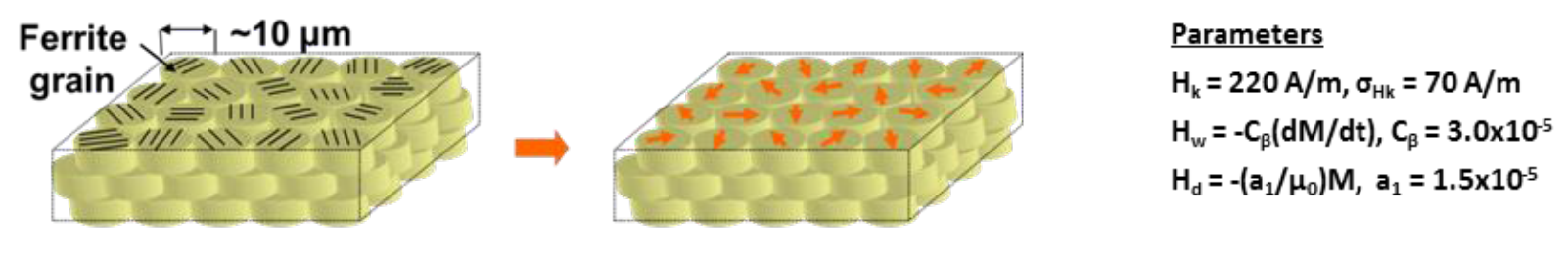

Fig. 4 A grain magnetics model and a single set of parameters for $\mathrm{Ni}-\mathrm{Zn}$ ferrite. 
As shown in Fig. 5, B-H major and minor loops are well reproduced by this simple model. The effective magnetic fields ( $\left.H_{\text {eff }}\right)$ for the LLG equation are the sum of $H_{\mathrm{k}}, H_{\mathrm{w}}, H_{\mathrm{d}}$, and the external field $\left(H_{\mathrm{ext}}\right)$. Once we determine a single set of the parameters for those fields (Fig. 4) with a dc major loop, minor loops with various $B_{\mathrm{m}}$ at all the frequencies are in good agreement with those obtained by the experiment. Briefly, here $H_{\mathrm{k}}$ and $H_{\mathrm{d}}$ determine coercivity and slopes near coercivity, respectively. Frequency dependence is decided by $H_{\mathrm{w}}$. More importantly, this model can reproduce dc-biased minor loops with various $H_{\mathrm{dc}}$ quite well. When we increase the dc bias, the loops become stretched and asymmetric: all these characteristic features are well reproduced as shown in Fig. 6.
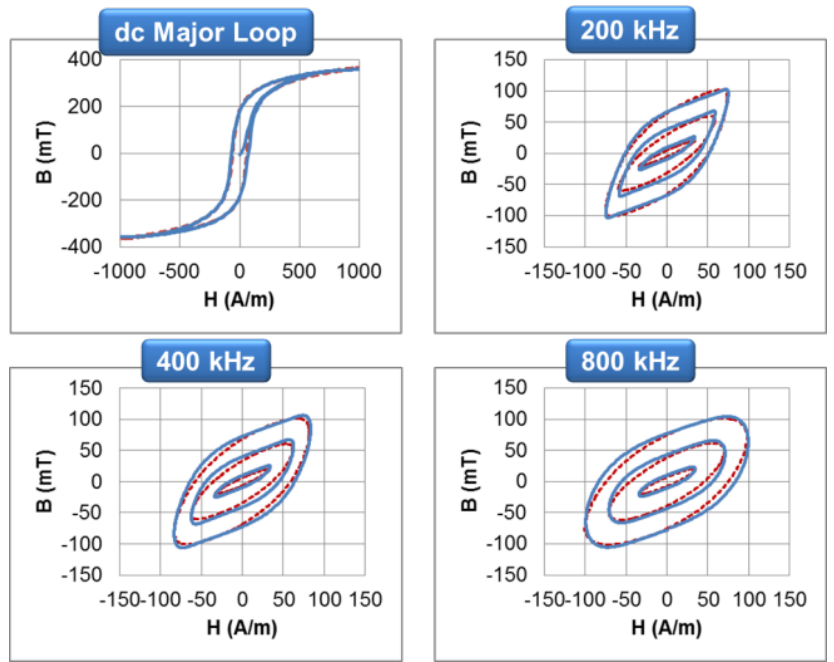

Fig. 5 Experimental (dashed lines) and simulation (solid lines) results of $B$ - $H$ loops without de bias.
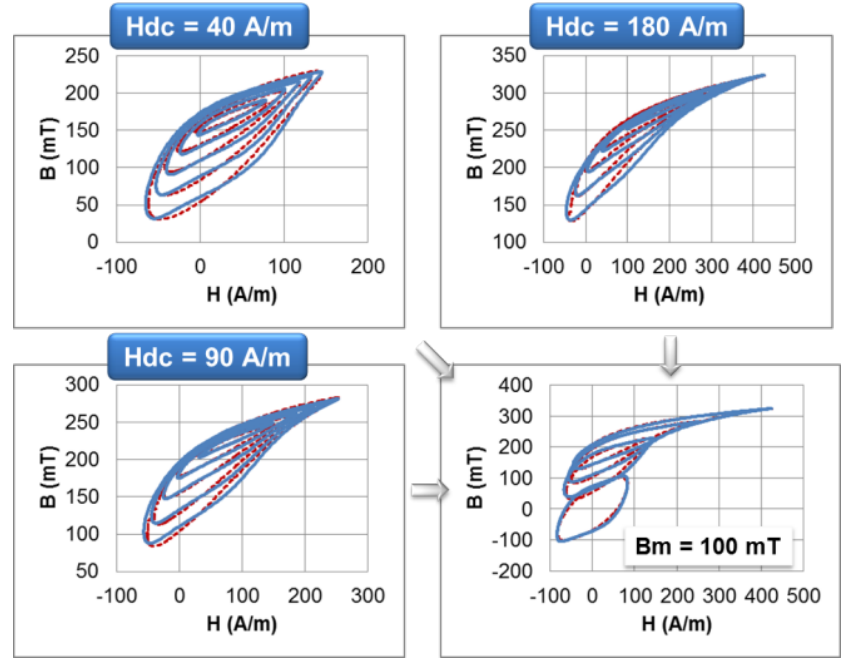

Fig. 6 Experimental (dashed lines) and simulation (solid lines) results of $B$ - $H$ loops with dc bias at $400 \mathrm{kHz}$.

\section{FINITE ELEMENT ANALYSIS WITH THE HYSTERESIS MODEL}

Integration of FEA with a hysteresis model is indispensable to directly calculate the core loss of inductors with arbitrary shapes. Former methods of FEA with hysteresis models, however, do not always provide satisfactory accuracy. In this study, we have developed a simulator for FEA integrated with the GM model. The calculation procedures for the simulator are as follows: From a given field $H$, we calculate magnetization $M$ of each element using the hysteresis model. Next, from the calculated $M$, we derive new $H$ of the elements with the Maxwell equations using FEA. The new $H$ is then given again to the hysteresis model for the calculation of new $M$. We compute these calculations repeatedly until all these values converge.

We perform both experiment and simulation for a wirewound inductor of the same Ni-Zn ferrite material (Fig. 7). Figure 8 illustrates snapshots of magnetic flux density and current density. The results of the inductance and core loss under dc bias are presented in Fig. 9. The current is the sum of dc current $I_{\mathrm{dc}}$ and ac current $i$, and the peak to peak amplitude of $i$ is 1 A here. The FEA simulation is performed for $400 \mathrm{kHz}$ and $800 \mathrm{kHz}$. As shown in Fig. 9, using the FEA with the GM model, we could directly obtain both the inductance and core loss values close to the experimental data.
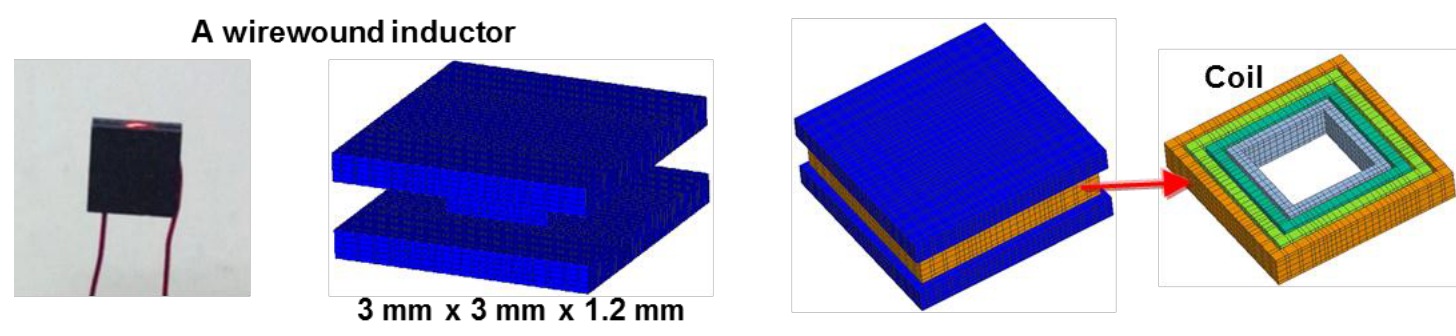

Fig. 7 A picture and a FEA model for a wirewound inductor and a coil. 
Finally, we should note some future prospects. The FEA with the GM model still requires much calculation time, and parallel computation should be explored for practical device design. Since our simulation allows arbitrary current waveforms, more general distorted waveforms with dc bias should be studied. Improvement of the models both for hysteresis and FEA is also necessary for better agreement of the results, and is currently under investigation.

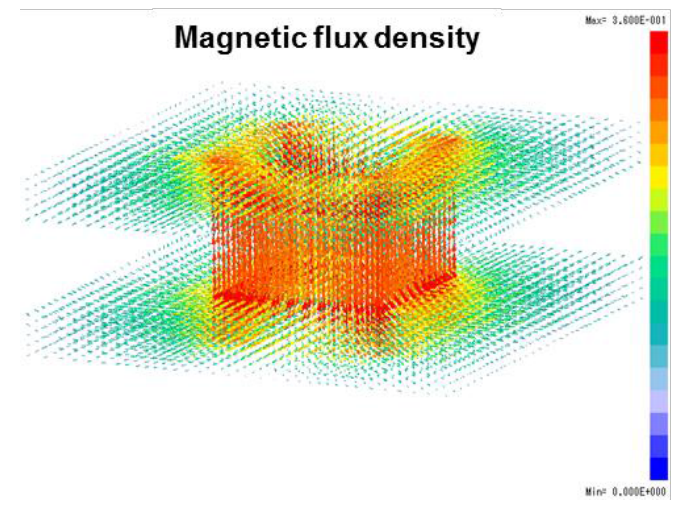

\section{Current density}

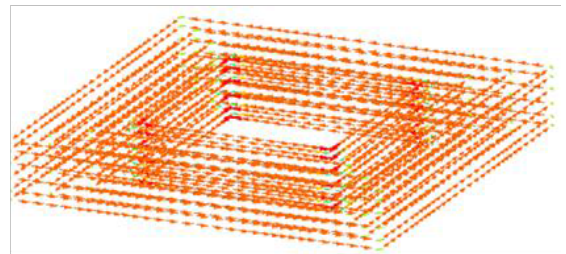

Fig. 8 Snapshots of magnetic flux density and current density.

Experiment

Inductance

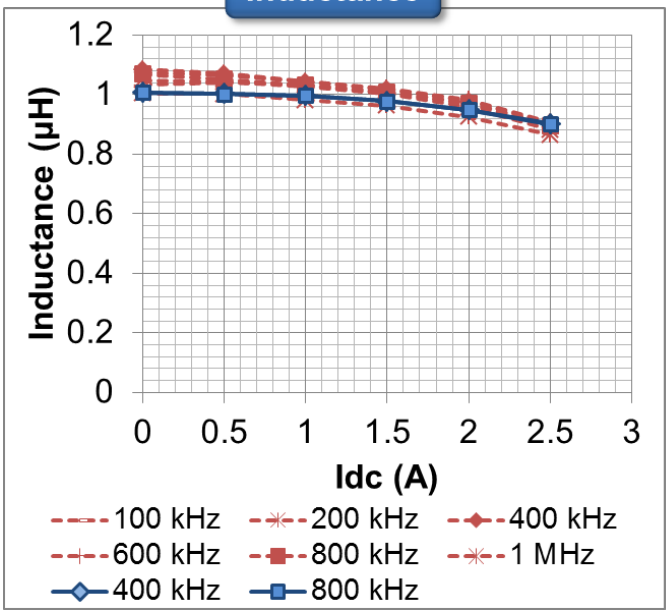

Finite Element Analysis

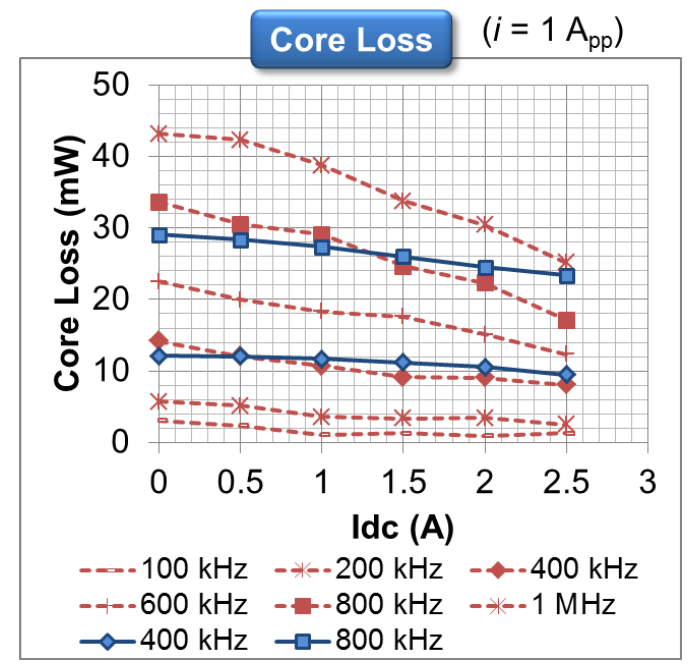

Fig. 9 Experimental (dashed lines) and FEA (solid lines) results of the inductance and core loss under dc bias current $I_{\mathrm{dc}}$.

\section{REFERENCES}

[1] C. A. Baguley, B. Carsten, and U. K. Madawala, IEEE Trans. Magn. 44, 246 (2008).

[2] J. Mühlethaler, J. Biela, J. W. Kolar, and A. Ecklebe, IEEE Trans. Power Electron. 27, 953 (2012).

[3] K. Kawano, M. Hachiya, Y. Iijima, N. Sato, and Y. Mizuno, J. Magn. Magn. Mater. 321, 2488 (2009).

[4] F. Preisach, Z. Phys. 94, 277 (1935).

[5] D. C. Jiles and D. L. Atherton, J. Magn. Magn. Mater. 61, 48 (1986).

[6] E. C. Stoner and E. P. Wohlfarth, Phil. Trans. Roy. Soc. 240A, 599 (1948).

[7] H. Kronmüller and M. Fähnle, "Micromagnetism and Microstructure of Ferromagnetic Solids", Cambridge University Press, Cambridge, U.K. 2003.

[8] D. L. Atherton and J. R. Beattie, IEEE Trans. Magn. 26, 3059 (1990).

[9] Y. Uehara, A. Furuya, J. Fujisaki, K. Shimizu, H. Oshima, M. Suzuki, The 34th Annual Conference on Magnetics in Japan, 6pC-4 (2010). 